\title{
Salvage pneumonectomy after definitive chemo-radiotherapy
}

\author{
Luca Bertolaccini ${ }^{1}$, Lorenzo Spaggiari ${ }^{1,2}$ \\ ${ }^{1}$ Division of Thoracic Surgery, IEO, European Institute of Oncology IRCCS, Milan, Italy; ${ }^{2}$ Department of Oncology and Hemato-Oncology, \\ University of Milan, Milan, Italy \\ Contributions: (I) Conception and design: L Bertolaccini; (II) Administrative support: L Spaggiari; (III) Provision of study materials or patients: \\ L Spaggiari; (IV) Collection and assembly of data: L Bertolaccini; (V) Data analysis and interpretation: All authors; (VI) Manuscript writing: All \\ authors; (VII) Final approval of manuscript: All authors. \\ Correspondence to: Luca Bertolaccini, MD, PhD, FCCP. Division of Thoracic Surgery, IEO, European Institute of Oncology IRCCS, Via Ripamonti \\ 435, 20141 Milan, Italy. Email: luca.bertolaccini@gmail.com.
}

\begin{abstract}
Guidelines and recommendations for not small cell lung cancer in stage IIIA include induction chemoradiotherapy followed by surgical resection, induction chemotherapy followed by surgery, and definitive chemoradiotherapy. After definitive high dose chemoradiation, $>35 \%$ of patients will locally relapse. In a slight proportion of patients, the recurrent disease is isolated local relapse not suitable for curative-intent radiotherapy. Therefore, complete resection (salvage surgery) is the first curative-intent modality of treatment. Salvage surgery is technically feasible in high-volume referral centres when indicated. Even if salvage surgery is a challenging operation, can be performed with adequate morbidity, mortality, and long-term outcomes, even when anatomical resections larger than a lobectomy or extended resections are necessary. The prognostic analyses of salvage surgery could offer a background for further discussion of the factors determinant to undertake this strategy. Strictly multidisciplinary meetings are crucial to deciding on salvage lung resection. Specific attention should be paid to the recurrence location of original cancer. The task remains in identifying patients promptly with persistent tumour after prior treatment and with local failure. This careful selection and the surgical expertise allow for successful salvage pneumonectomy with acceptable morbidity.
\end{abstract}

Keywords: Lung cancer; pneumonectomy; salvage surgery; advanced stage

Received: 17 October 2019; Accepted: 14 November 2019; Published: 10 April 2020.

doi: $10.21037 /$ shc.2019.11.07

View this article at: http://dx.doi.org/10.21037/shc.2019.11.07

\section{Introduction}

Guidelines and recommendations for not small cell lung cancer in stage IIIA include induction chemoradiotherapy followed by surgical resection, induction chemotherapy followed by surgery, and definitive chemoradiotherapy (1). After definitive high dose chemoradiation, $>35 \%$ of patients will locally relapse. In a slight proportion of patients, the recurrent disease is isolated local relapse not suitable for curative-intent radiotherapy. Therefore, complete resection (salvage surgery) is the first curative-intent modality of treatment (2). Previous studies demonstrated an acceptable safety without the statistical power to detect associations between prognosis and salvage surgery $(3,4)$.

\section{The definition of salvage thoracic surgery}

Salvage surgery is the surgical resection of recurrent or persistent primary lung tumours after preceding local treatments (5). The most frequent indications for a salvage operation are apparent relapse by computed tomography (CT), continually irregular positron emission tomography (PET) discoveries after the radiotherapy conclusion, and a postponed choice to convert to a trimodal approach (2). However, on a broader reason, salvage surgery could be described as surgical resection of recurrent or persistent primary lung tumours after previous local treatments, as well to urgent symptoms such as haemoptysis (6). The indications for salvage lung resection can be summarised as 
a biopsy-proven NSCLC before any treatment, underwent curative-intent radiotherapy with or without chemotherapy or a trimodally treatment not incorporating surgical resection with a subsequent salvage pulmonary resection.

\section{Selection of the patients for the salvage surgery}

The selection of patients is one of the most crucial aspects to obtain positive effects of salvage surgery. FDG PET and magnetic resonance of the brain are essential for completing the staging. A new histological confirmation of the neoplasm is mandatory before discussing the indication. In patients with radiological findings of presumed lymph node involvement, Endobronchial ultrasound (EBUS) bronchoscopy should be performed. For more accurate staging, further advances in diagnostic imaging and development of new modalities are expected in the future. Assessment of circulating tumour cells could have an essential role in defining if salvage surgery is mandatory since circulating tumour cells (dissemination of the disease at the cellular level), were substantially associated with shorter progression-free survival and overall survival (7). Therefore, the selection criteria for salvage surgery could be:

(I) Early age ( $<70$ years) fit patients with a careful cardiorespiratory evaluation showing the feasibility of surgery;

(II) A patient determined and wholly informed about the proposed operation;

(III) Failure of previous surgical and medical treatments;

(IV) No other helpful treatments accessible;

(V) No shreds of evidence of extrathoracic disease (8).

Various methods of restaging after induction have been evaluated, and the results differ subject on the option. Restaging only by imaging is inaccurate due to high false negative and positive rates. Survival for patients, not downstage after induction, have satisfactory rate, like the downstage patient (4). The indication of salvage surgery should be discussed inside a multidisciplinary tumour. Contribution of thoracic surgeons in the assessment of treatment strategy during the multidisciplinary tumour board may change the entire treatment regimen.

\section{Surgical and postoperative tips and tricks}

In published series of salvage surgery, the number of patients is inadequate to define the early and longterm effects, since indications remain uncertain. The complexity of the procedures, the high morbidity and mortality associated in the postoperative period, and the need of a highly experienced team are some of the issues that may explain these small numbers. Two issues transform salvage surgery in a challenging procedure: the high doses of radiotherapy administered and the time between finishing radiotherapy treatment and resection ( $>8$ weeks). Concerning the reduced experience with salvage lung surgery and the small number of thoroughly selected patients, the perioperative risk appears not higher if compared with trimodally therapy models, even though the application of high radiation dose upfront the surgical operation. The more frequent complications were pneumonia, bronchopleural fistula, acute respiratory distress syndrome (ARDS), and recurrent laryngeal nerve palsy (mainly the left). ARDS develops commonly and is a significant cause of postoperative death. A coverage of bronchial stump with vascularized tissue must be taken into consideration to reduce the complication rate. Besides, a pleural decortication and tenting should minimise the remaining air space and air leakage. The recurrent laryngeal nerve injury should be avoided, to ensure expectoration and to sustain early postoperative mobilisation. The risk of pneumonia and post-resection empyema could be decreased by the administration of intravenous antibiotics, specifically during the first five postoperative days $(9,10)$. These complications, however, commonly developed concerning the length and the complexity of surgery and is also joint in surgery after induction chemoradiotherapy. Desmoplastic reactions due to preoperative radiotherapy and/or chemotherapy could worsen wound healing and give weaknesses to the anastomotic sites and bronchial stump (3).

\section{Brief review of the principal experiences reported in the literature}

A series of 40 NSCLC patients who underwent surgical resection after definitive chemo-radiotherapy was published by Sonnet in 2004. The median time between radiotherapy and surgery was two months (11). No postoperative mortality was reported due to a different radiation technique, a better selection of patients, the coverage of the bronchial stump and enhancements in the postoperative management. In 2008, Bauman et al. reviewed 24 patients underwent salvage surgery after definitive chemoradiotherapy with a median overall survival of 30 months (2). Thirty-five patients who underwent salvage resection after definitive chemoradiotherapy were 
published by Casiraghi et al. The series comprised patients who received <59 Gy with an extended period between radiotherapy and surgery, and patients who received $>59$ Gy where surgery was performed $<8$ weeks after finishing radiotherapy with a $51 \%$ morbidity and $5.7 \%$ mortality rates. The morbidity was correlated with $>3$ chemotherapy cycles, but not related to a higher dose of radiotherapy or more extended interval between the end of radiotherapy and surgery (12). Uramoto et al. found a 5-year overall survival rates in salvage surgery patients of $40.4 \%$, demonstrating a favourable long-term outcome of salvage surgery (5). In the series reported by Dickhoff $e t a l$., the overall survival was 46 months (13).

\section{Discussion}

Salvage lung surgery is technically challenging due to the intraoperative risks of major vessel injury and the high rates of significant morbidity and mortality. Outstanding surgical outcomes can be justified by numerous potential factors. This cohort represented a highly selected group of patients with favourable response to their initial treatments and functional performance status who were expected to benefit from the surgical alternative based on a combination of objective and independent information. Patients with an ineffective response to preoperative treatments were excluded. Advancements in radiotherapy and the introduction of a wide variety of new-generation agents provided concurrently better compliance and efficacy. The delay intrinsic in salvage surgery may enhance intraoperative risk, but confirmation of the nonappearance of distant recurrences is essential to prevent unnecessary resection. Lastly, induction therapy followed by lung surgery has often been used in stage III disease patients. Clinical experiences and improved perioperative care, as in high-volume referral centres, should provide to decreased rates of mortality and morbidity in salvage surgery. Recognition of proper candidates who will benefit from salvage surgery has not been still explained and has been tough. Some studies of neoadjuvant therapy have described that nodal sterilisation and local therapeutic effect have been associated with extended survival (14).

There are more than a few limitations related to this narrative review. All data were retrospectively collected. Furthermore, due to the small sample sizes, caution should be used in the interpretation of the results. Prospective studies in larger cohorts at various institutions will be needed to explain the efficacy of salvage surgery and to overcome these limitations. A comprehensive examination of a prospective trial could produce current information about the relationships between the survival and timing of surgery and the patient selection, along with the therapeutic consequences in pathological specimens.

\section{Conclusions}

Salvage surgery is technically achievable when indicated and could be achieved with acceptable morbidity, mortality, and long-term outcomes, even when a pneumonectomy is required (15). The prognostic analyses of salvage surgery could offer a background for further discussion of the factors determinant to undertake this strategy. Strictly multidisciplinary meetings are crucial to deciding on salvage lung resection. Specific attention should be paid to the recurrence location of original cancer. The task remains in identifying patients promptly with persistent tumour after prior treatment and with local failure. This careful selection and the surgical expertise allow for successful salvage pneumonectomy with acceptable morbidity.

\section{Acknowledgments}

None.

\section{Footnote}

Conflicts of Interest: The authors have no conflicts of interest to declare.

Ethical Statement: The authors are accountable for all aspects of the work in ensuring that questions related to the accuracy or integrity of any part of the work are appropriately investigated and resolved.

\section{References}

1. Aupérin A, Le Péchoux C, Rolland E, et al. Meta-Analysis of Concomitant Versus Sequential Radiochemotherapy in Locally Advanced Non-Small-Cell Lung Cancer. J Clin Oncol 2010;28:2181-90.

2. Bauman JE, Mulligan MS, Martins RG, et al. Salvage Lung Resection After Definitive Radiation (>59 Gy) for Non-Small Cell Lung Cancer: Surgical and Oncologic Outcomes. Ann Thorac Surg 2008;86:1632-9.

3. Sonobe M, Yutaka Y, Nakajima D, et al. Salvage surgery after chemo- or chemoradiotherapy for initially 
unresectable lung carcinoma. Ann Thorac Surg 2019. [Epub ahead of print].

4. Romero-Vielva L, Viteri S, Moya-Horno I, et al. Salvage surgery after definitive chemo-radiotherapy for patients with Non-Small Cell Lung Cancer. Lung Cancer 2019;133:117-22.

5. Uramoto H, Nakajima $Y$, Kinoshita $H$, et al. Equivalent Outcome of Patients with Locally Advanced NSCLC Treated with Salvage Surgery Compared to Induction Chemotherapy Followed by Surgical Resection. Anticancer Res 2016;36:4243-7.

6. Uramoto H. Current Topics on Salvage Thoracic Surgery in Patients with Primary Lung Cancer. Ann Thorac Cardiovasc Surg 2016;22:65-8.

7. Nakanishi K, Mizuno T, Sakakura N, et al. Salvage surgery for small cell lung cancer after chemoradiotherapy. Jpn J Clin Oncol 2019;49:389-92.

8. Presicci PF, Veronesi G, D'Aiuto M, et al. Long-term survival after salvage surgery for colorectal lung metastases. Ann Thorac Surg 2005;79:325-6.

9. Schreiner W, Dudek W, Lettmaier S, et al. Long-Term Survival after Salvage Surgery for Local Failure after Definitive Chemoradiation Therapy for Locally Advanced Non-small Cell Lung Cancer. Thorac Cardiovasc Surg

doi: 10.21037/shc.2019.11.07

Cite this article as: Bertolaccini L, Spaggiari L. Salvage pneumonectomy after definitive chemo-radiotherapy. Shanghai Chest 2020;4:14.
2018;66:135-41.

10. Antonoff MB, Correa AM, Sepesi B, et al. Salvage pulmonary resection after stereotactic body radiotherapy: A feasible and safe option for local failure in selected patients. J Thorac Cardiovasc Surg 2017;154:689-99.

11. Sonett JR, Suntharalingam M, Edelman MJ, et al. Pulmonary Resection After Curative Intent Radiotherapy (>59 Gy) and Concurrent Chemotherapy in Non-SmallCell Lung Cancer. Ann Thorac Surg 2004;78:1200-5.

12. Casiraghi M, Maisonneuve P, Piperno G, et al. Salvage Surgery After Definitive Chemoradiotherapy for Nonsmall Cell Lung Cancer. Semin Thorac Cardiovasc Surg 2017;29:233-41.

13. Dickhoff C, Dahele M, Paul MA, et al. Salvage surgery for locoregional recurrence or persistent tumor after high dose chemoradiotherapy for locally advanced non-small cell lung cancer. Lung Cancer 2016;94:108-13.

14. Shimada Y, Suzuki K, Okada M, et al. Feasibility and efficacy of salvage lung resection after definitive chemoradiation therapy for Stage III non-small-cell lung cancer. Interact Cardiovasc Thorac Surg 2016;23:895-901.

15. Kaba E, Ozyurtkan MO, Ayalp K, et al. Salvage thoracic surgery in patients with lung cancer: potential indications and benefits. J Cardiothorac Surg 2018;13:13. 